\title{
An Audit of the Outcomes of Thymic Surgery
}

\author{
Pralaya Khadka, Bibhusal Thapa, Ranjan Sapkota, Aakriti Sharma and Prakash Sayami \\ Department of Thoracic Surgery, Manmohan Cardiothoracic Vascular and Transplant Center, Maharajgunj, Kathmandu, Nepal
}

\begin{abstract}
Most pathology of the thymus gland warrant its surgical removal; and this requires significant expertise and adequate medical set-up. This study aimed to audit the results of thymectomies performed in a specialised tertiary level centre in a resource-poor country. The outcomes of open and minimally invasive video-assisted thoracoscopic surgery (VATS) thymectomies were also compared. Out of 58 patients operated at Department of Thoracic Surgery, Manmohan Cardiothoracic Vascular and Transplant Center, Maharajgunj, Kathmandu, Nepal between October 2012 and January 2019, 33 patients underwent open thymectomy and 25 had VATS thymectomy. We conducted a retrospective search to look at operative time, blood loss and length of postoperative hospitalisation. The open surgery group was followed for an average of 32 months, and the VATS cohort for 38 months. No significant differences were found in mean operative times and overall survival between groups. In selected cases, thymectomy via VATS is safer and comparable to open thymectomy in terms of operative safety and oncological completeness.
\end{abstract}

Key Words: Thymus, VATS, Thymectomy, Outcome.

How to cite this article: Khadka P, Thapa B, Sapkota R, Sharma A, Sayami P. An Audit of the Outcomes of Thymic Surgery. J Coll Physicians Surg Pak 2021; 31(05):602-604.

The most common indications for the removal of the thymus gland are thymoma with or without myasthenia gravis and nonthymomatous myasthenia gravis. There are various well-established approaches to remove the thymus surgically, namely transthoracic, transcervical, video-assisted thoracoscopic, and robotic-assisted thoracoscopic thymectomy. ${ }^{1}$ Median sternotomy is the most common route used to surgically excise the gland. ${ }^{2}$ Over the past decade, minimally invasive approaches have gained popularity due to purported better cosmesis, lower morbidity, and early recovery. ${ }^{3}$

The objectives of this study were to audit the results of thymectomy at our centre, and to compare the early outcomes of thymectomy via video-assisted thoracoscopic surgery (VATS) and sternotomy in Nepalese patients in terms of operative safety, morbidities, mortality, and oncologic safety.

After obtaining Institutional Review Board approval (IRB Ref. No. 20/076/77), database search was conducted for patients who had thymic surgery between October 2012 and January 2019. The choice of approach to thymectomy was based on a number of factors including size of the tumour, patient preference, and the operative experience of the surgeon involved. In general, VATS approach was undertaken for tumors smaller than $5 \mathrm{~cm}$.

Correspondence to: Dr. Pralaya Khadka, Department of Thoracic Surgery, Manmohan Cardiothoracic Vascular and Transplant Center, Maharajgunj, Kathmandu, Nepal

E-mail: pralayakhadka@gmail.com

Received: August 08, 2020; Revised: December 18, 2020;

Accepted: December 28, 2020

DOI: https://doi.org/10.29271/jcpsp.2021.05.602
VATS was done via the standard 3-port techniques with patient at 30 degrees tilt. Thoracoscopic port was created in the $8^{\text {th }}$ ICS in MAL, in addition to two working ports, one in the $3^{\text {rd }}$ ICS in MAL and another in the $5^{\text {th }}$ ICS in AAL. Total, radical or extended thymectomy was done, in each instance.

Controlled variables included age, gender, presence of myasthenia and its type (thymomatous and nonthymomatous), preoperative diagnosis, and Masaoka stage for thymoma. Variables observed were operative time, amount of blood loss, length of hospital stay, complications and survival rates. For statistical analysis, SPSS software version 20.0 (SPSS Institute, Chicago, IL) was utilised. Qualitative data were expressed as frequencies and percentages and quantitative data were expressed as mean \pm S.D. and median (IQR). Continuous variables like operative time, blood loss and hospital stay were compared using independent sample t-test and follow-up by Mann-Whitney test and categorical variable, like mortality, was compared using Chi-square test/Fischer's Exact test. A p $<0.05$ was considered significant.

A total of 85 patients had undergone thymectomy. All patients aged 16 years and above were included. Twenty-seven cases were excluded from the study for reasons including nonthymic pathologic diagnoses, loss of data from database, and loss to follow-up. The remaining 58 patients were grouped them into two cohorts, VATS and open. Thirty-three (56.9\%) patients had had open thymic removal via sternotomy; whereas, 25 (43.1\%) had undergone VATS thymectomy. There was a slight female preponderence in both groups; and patients undergoing VATS were younger (Table I). Most with myasthenia gravis underwent VATS. In the VATS group, $20(80 \%)$ had myasthenia as compared to only $8(24.2 \%)$ in the open group. Forty-one 
patients had thymoma, of which $22(53.7 \%)$ were Masaoka stage I and 7 (17\%) stage IIA, 6 (14.6) stage IIB, and 6 (14.6\%) stage III.Sixteen (39.0\%) thymomas wereassociated with myasthenia gravis. Conversely, out of the total 28 myasthenia patients, $5(17.9 \%)$ were found to have thymoma histologically.

Table I: Comparison of variables in both groups.

\begin{tabular}{|c|c|c|c|}
\hline & Sternotomy & VATS & p-value \\
\hline Number & 33 & 25 & - \\
\hline Age & $41.2 \pm 19.0$ & $38.1 \pm 14.1$ & 0.499 \\
\hline $\begin{array}{l}\text { Gender } \\
{[(\mathrm{M}(\%) / \mathrm{F}(\%)]}\end{array}$ & $15(45.5) / 18(54.5)$ & $12(48) / 13(52)$ & 0.847 \\
\hline MG (\%) & $8(24.2)$ & $20(80)$ & $<0.001$ \\
\hline LoS (days) & $7.0 \pm 3.8$ & $5.3 \pm 2.9$ & 0.073 \\
\hline Tube drainage (days) & $3.3 \pm 1.1$ & $2.7 \pm 1.7$ & 0.102 \\
\hline $\begin{array}{l}\text { Surgery duration } \\
\text { (min) }\end{array}$ & $190 \pm 28$ & $187 \pm 24.5$ & 0.671 \\
\hline Blood loss (ml) & $219.7 \pm 69.5$ & $135.2 \pm 38.5$ & $<0.001$ \\
\hline Conversion rate (\%) & & $1(4)$ & - \\
\hline $\begin{array}{l}\text { Follow-up median } \\
\text { [mo(IQR)] }\end{array}$ & $32(19-65.5)$ & $38(2.5-65)$ & 0.596 \\
\hline Mortality (\%) & $1(3)$ & $0(0)$ & $>0.999$ \\
\hline 3y-SR (\%) & $32(97)$ & $25(100)$ & \\
\hline
\end{tabular}

Table II: Subgroup analysis for patients with thymoma.

\begin{tabular}{|l|c|c|c|}
\hline & Sternotomy & VATS & p-value \\
\hline Number & 29 & 12 & - \\
\hline Age & $41.2 \pm 20.0$ & $45.3 \pm 15.2$ & 0.523 \\
\hline Myasthenia (\%) & $6(20.7)$ & $2(16.7)$ & $>0.999$ \\
\hline Operative time & $187.2 \pm 26.3$ & $185.4 \pm 23.6$ & 0.836 \\
\hline Blood loss & $213.8 \pm 70.6$ & $134.2 \pm 42.3$ & 0.001 \\
\hline Length of stay & $6.2 \pm 2.5$ & $5.3 \pm 1.6$ & 0.272 \\
\hline Mortality (\%) & $0(0)$ & $0(0)$ & - \\
\hline Recurrence (\%) & $1(3.4)$ & $0(0)$ & $>0.999$ \\
\hline Size of tumor (Cm) & $7.9 \pm 2.7$ & $4.9 \pm 1.2$ & 0.001 \\
\hline Negative margins (\%) & $29(100)$ & $12(100)$ & - \\
\hline
\end{tabular}

The mean operative durations of both groups were similar, 190 \pm 28 minutes for sternotomy and $187 \pm 24.5$ minutes for VATS $(p=0.671)$. Although average blood loss in VATS was moderately less, $135.2 \pm 38.5 \mathrm{ml}$ versus $219.7 \pm 69.5 \mathrm{ml}$ in sternotomy, the difference was significantly statistical $(p<0.001)$. One of the VATS procedure had to be converted due to uncontrollable bleeding from the junction of the left brachiocephalic vein and superior vena cava, as a result of inadvertent injury. Average chest intube duration was $3.3 \pm 1.1$ days for sternotomy, and $2.7 \pm 1.7$ days for VATS $(p=0.102)$. The hospital stay for sternotomy group was longer than VATS cohort $(7.0 \pm 3.8$ days vs. $5.3 \pm 2.9$ days); but it was not statistically significant ( $p$ $=0.073$ ). In other words, those who underwent VATS could be discharged about 2 days earlier on average as compared to those who underwent sternotomy, implying earlier postoperative recovery. When the length of stay of myasthenia patients were compared with non-myasthenics, the difference was not found to be statistically significant $(6.8 \pm 4.4$ days vs. $5.8 \pm 2.3$ days; $p=0.268$ ).

Among 28 patients with myasthenia, 20 (71.4\%) underwent VATS thymectomy and 8 (28.6\%) underwent thymic removal via sternotomy. All patients were stable prior to operation. One patient in the VATS group was given plasmapheresis two days before the procedure. Post-procedure in the VATS cohort, two (10\%) could be taken off medication completely. In $12(60 \%)$ patients, the dose of medications could be reduced; whereas, in 6 (30\%) patients, the preoperative doses were maintained up to three years after operation. In the sternotomy group, the dose of medications could be reduced in 4 patients $(50 \%)$ and three patients (37.5\%) were taking the same doses at 3-year follow-up. One patient (12.5\%) in this group could be taken off medications at 6-month follow-up.

Six myasthenia patients developed crisis, three in VATS group and three in the sternotomy group. One of the patients in thesternotomy group, who developed myasthenic crisis, succumbed. Surgical margins were demonstrated to be negative in all patients with thymoma ( 29 open and 12 VATS). There were no sternotomy-related complications seen postoperatively. There was a recurrence of thymoma in the sternotomy group at the 2year follow-up as evidenced by CT-guided biopsy. The patient had Masaoka stage III thymoma and deferred postoperative radiation therapy (Tablell).

Thymectomy is a significant operative undertaking, requiring significant operative expertise and considerable perioperative medical management; therefore, limiting this service to limited specialised hospitals. It is common knowledge that such expertise required training; and the facilities are not readily available in resource-poor countries, like Nepal.

Despite our limitations, this audit of thymectomies conducted in our centre demonstrated good patient outcomes with limited morbidity and acceptable mortality.

In terms of age and female preponderance of thymomas, our results mirror that of western literature. About $18 \%$ of the myasthenic patients had a concurrent thymoma; and myasthenia was associated in $39 \%$ of patients with thymoma, which is not very different from what is previously reported. ${ }^{4}$

Recent individual large cohort reports and meta-analyses comparing VATS thymectomies with open thymectomies have unequivocally established their procedural and oncological safety. ${ }^{5}$ They have also been shown to result in lesser blood loss, shorter operative times, lesser pain and quicker discharges. ${ }^{6}$ However, it is also known that VATS thymectomies do have significant learning curves. Our early experience with VATS thymectomies show similar results with the operative time, conversion rates as reported in literature. The authors were able to excise tumours up to $6 \mathrm{~cm}$ via VATS, and the surgical margins were found to be negative in all the patients. The follow-up in these patients is relatively short and although there have not been significant differences in recurrences between groups in our cohort, a longer follow-up will be required to truly 
assessthis.

In terms of thymic surgery for myasthenia, it was seen that $50-60 \%$ of patients required less doses of their medications postoperatively. This can have implications on the reduction of possible side effects of the immusuppressants. In addition, about one-tenth of patients could be taken off their medications completely. These were results seen up to a 3-year follow-up period.

VATS thymectomies, when done in selected patients, have delivered good results with the added advantages of smaller scars and quicker recovery in our centre. With accumulating experience, we have been able to achieve results comparable to those fromlarger, more resourced centres.

\section{ETHICALAPPROVAL:}

The Institutional Review Board of Manmohan Cardiothoracic Vascular and Transplant Center, Institute of Medicine, Tribhuvan University approved the study (Ref. No. 20/076/77).

\section{CONFLICT OF INTEREST:}

The authors declared no conflict of interest.

\section{AUTHORS' CONTRIBUTION:}

PK: Data collection, analysis and drafting.

BT: Concept and editing.

RS: Data analysis and editing.

AS: Data collection and analysis.
PS: Concept, revision and final approval.

\section{REFERENCES}

1. Daniel VC, Wright CD. Extended transsternal thymectomy. Thorac Surg Clin 2010; 20(2):245-52. doi: 10.1016/j. thorsurg. 2010.02.005.

2. Deeb ME, Brinster CJ, Kucharzuk J, Shrager JB, Kaiser LR. Expanded indications for transcervical thymectomy in the management of anterior mediastinal masses. Ann Thorac Surg 2001; 72(1):208-11. doi: 10.1016/s0003-4975(01) 02675-3.

3. Gkouma A. Robotically assisted thymectomy: A review of the literature. J Robot Surg 2018; 12(1):3-10. doi: 10.1007/ s11701-017-0748-3.

4. Tianci C, Shen Z, Chen S, Lin Y, Gao L, Zhang Z, et al. Median sternotomy versus minimally invasive thymectomy for early-stage thymoma. Medicine 2019; 98(51). doi: 10.1097/MD.0000000000018359.

5. Gilhus NE, Verschuuren J. Myasthenia gravis: subgroup classification and therapeutic strategies. Lancet Neurol 2015; 14(10):1023-36. doi: 10.1016/S1474-4422(15) 00145-3.

6. Yang $Y$, Dong J, Huang Y. Thoracoscopic thymectomy versus open thymectomy for the treatment of thymoma: $A$ meta-analysis. Eur J Surg Oncol J Eur Soc Surg Oncol Br Assoc Surg Oncol 2016; 42(11):1720-8. doi: 10.1016/ j.ejso.2016.03.029. 